\title{
" Il faut que je périsse ou que je me venge ». Politiques de la méchanceté dans les Lettres de Cyrano
}

\author{
Alex Bellemare \\ Université de Montréal, Sorbonne Nouvelle Paris 3
}

Cyrano de Bergerac, figure libertine dont l'œuvre bigarrée s'inscrit volontiers dans une dynamique de disputes et de conflits, publie en 1654, dans une variante amendée et dépouillée des passages les plus scandaleux présents dans le manuscrit original, un recueil de Lettres satiriques et amoureuses, ouvrage ayant pour principal moteur la médisance et l'insulte. CEuvre plusieurs fois caviardée, sans arrêt soumise à des procédures de correction ou de censure, l'auteur supprimant ici des passages jugés trop outrageants, l'éditeur 
ajoutant là des propos parfaitement orthodoxes pour chasser tout soupçon d'impiété, les Lettres déclinent dans une variété de tons et de registres les griefs les plus multiples ${ }^{1}$. Si les cibles de Cyrano sont disparates et nombreuses, les charges les plus âcres sont par contre dirigées vers des écrivains contemporains, anciens amis et nouvellement rivaux. En effet, la plupart des lettres satiriques sont adressées à une figure reconnaissable que Cyrano entend déconstruire jusqu'à la caricature. Ce faisant, les Lettres construisent un dispositif énonciatif retors, polémique, et dont la visée apparaît parfois fuyante: par le recours à différentes figurations de la méchanceté, elles mettent en œuvre la force performative de la parole ulcérée. La méchanceté de Cyrano prend ainsi deux directions principales: d'une part, elle constitue un mode particulièrement perverti d'interlocution, où la parole s'investit

1 L'histoire éditoriale des Lettres, pour le moins complexe et enchevêtrée, rend néanmoins compte des différentes stratégies de dissimulation et de prudence mises de l'avant par les écrivains libertins pour échapper au pouvoir censorial. Les Lettres, que Cyrano publie de son vivant en 1654, connaissent divers états et variantes : il existe en effet une version manuscrite (manuscrit coté Nouv. Acq. 4557 conservé à la Bibliothèque nationale de France), une édition originale imprimée et insérée dans un recueil d'œuvres multiples (Les OEuvres diverses de M. Cyrano de Bergerac, Paris, Charles de Sercy, 1654), une édition cartonnée (voir entre autres l'édition cotée $4^{\circ} \mathrm{BL}$ 5296 conservée à la Bibliothèque de l'Arsenal) et, enfin, une édition posthume (Les Nouvelles OEuvres de Cyrano de Bergerac, Paris, Charles de Sercy, 1662). Paradoxalement, malgré la dimension dissidente et transgressive des écrits de Cyrano, son œuvre n'a jamais été mise à l'index. Mathilde Lévesque souligne que l'une des raisons pouvant expliquer cet étrange phénomène est l'usage récurrent de l'autocensure, qui consiste pour l'essentiel à varier jusqu'au brouillage les perspectives possibles: «Si les retouches sont multiples, elles sont toujours stratégiques, le plus souvent polyphoniques, laissent entendre, derrière la voix de la raison, celle de l'écrivain subversif. » (2009, p. 488) Le présent article s'intéressera moins aux effets de variance des différentes éditions entre elles qu'à la représentation de la méchanceté que les Lettres accueillent. Toutes les citations des Lettres renvoient à l'édition préparée par Jean-Charles Darmon et Alain Mothu. 
d'une constante aigreur et, d'autre part, elle témoigne d'une pathologie de la perception de l'autre et, ce faisant, d'une distorsion de l'affirmation de soi.

La dimension hautement offensante des Lettres a été perçue rapidement, malgré les précautions rhétoriques mises en œuvre par Cyrano pour les adoucir et se prémunir d'éventuels procès. Gabriel Guéret, critique littéraire contemporain de Cyrano, érigeait déjà les Lettres en " "amas d'ordures" qu'il avait répandus sans vergogne et sans pitié sur Dassoucy, Montfleury, Scarron » (Alcover, 1990, p. 2). La méchanceté des Lettres ne se réduit toutefois pas au simple règlement de comptes entre écrivains concurrents, mais s'inscrit plutôt dans un contexte spécifique où les libertins du XVIIe siècle, marginalisés et condamnés par la doxa politique et religieuse, sont suspects parce que faisant l'expérience d'une liberté apparemment sans borne à la fois dans leurs comportements scandaleux et dans leurs discours séditieux. La notion de liberté apparaît au cœur de la définition du libertinage, à telle enseigne que Jean-Pierre Cavaillé n'hésite pas à affirmer que "la grande affaire du "libertinage", comme cristallisation de conflits de tous ordres, est la "liberté" (la notion de liberté appréhendée négativement, évoquée spontanément et directement dans le mot) 》 (2012, p. 376). La méchanceté, dans cette perspective libertine, prend rapidement la forme d'exclusions systématiques: Cyrano écrit «contre» une masse de discours dominants considérés détestables parce que fondés sur des impostures. Les Lettres témoignent donc exemplairement d'une écriture en régime répressif: Cyrano, soupçonné d'irréligion, répond à ces accusations avec une violence analogue. Conformément à la logique souterraine qui définit l'expérience libertine comme une démultiplication d'épreuves construisant et déconstruisant leur 
identité, « les libertins apparaissent soit comme les produits de la violence, soit comme des acteurs, des praticiens de la violence intellectuelle » (Van Damme, 2007, § 10). Bref, la méchanceté apparaît comme une force impérieuse et réversible à l'envi, c'està-dire que la victime du méchant ne demeure jamais passive et épouse l'énergie négative et destructrice du persécuteur.

L'usage de la modalité épistolaire fait miroiter par ailleurs une communication impossible ou tronquée : la lettre, espace scripturaire privé et de relative liberté puisque sa réception est supposée confidentielle, permet de nommer toutes les tromperies et superstitions que les libertins répriment d'ordinaire dans la vie publique. Ainsi se met en place une tension entre le public et le privé, effet de mise à distance que le genre épistolaire accentue encore davantage. Cyrano, en s'exprimant avec violence contre des figures établies et des discours normatifs, construit aussi, en filigrane, une identité bariolée et bâtie selon une altérité constamment négative et agonistique. La dynamique épistolaire, qui suppose l'absence de celui à qui l'on s'adresse, renvoie aussi à une forme de fictionnalisation de soi : "L'autre auquel il écrit n'est plus l'autre réel mais celui qu'il imagine, et le moi qu'il met en scène à destination de l'autre est lui-même un moi imaginaire. » (Ferreyrolles, 2010, p. 14) Pour un écrivain polygraphe comme Cyrano, ayant pratiqué les genres les plus divers, le choix de figer ses «sautes d'humeur» dans la forme épistolaire, plutôt que dans un roman par exemple ${ }^{2}$, met en relief la dimension

\footnotetext{
2 Cyrano s'est surtout rendu célèbre dans l'histoire de la littérature pour son roman inachevé Les États et Empires de la Lune et du Soleil (1657-1662). «Trop longtemps délaissées » par la critique, Jean-Charles Darmon souligne que les Lettres sont des "voyages incongrus et rapides dans et par le langage » et qu'« elles dévoilent aussi, en ses réalités dérangeantes, un autre
} 
profondément relationnelle et dynamique du propos cyranien. C'est encore ce que remarque Simon Harel, qui suggère que "toute réflexion sur la méchanceté littéraire renoue avec la nécessité de mieux baliser les figures de l'autre en soi » (2008, p. 192). Effectivement, l'écriture de la colère constitue une performance ritualisée, une atteinte à autrui, une violence qui met en jeu l'efficace de la parole.

S'agissant de la lettre au XVIIe siècle, au moins trois instances distinctes mais solidaires sont mobilisées dans l'échange épistolaire: le destinataire et le destinateur, à l'évidence, mais également un "tiers inclus », une figure dont la présence est déclinée conventionnellement sous la forme d'allusions. À propos de la lettre familière au XVIII siècle, Benoît Melançon souligne qu'elle «convoque ainsi divers lecteurs et personnages dès son écriture, les représente, leur fait jouer plusieurs rôles, les compare les uns aux autres, les unit ou les sépare » (1996, p. 370). Dans le cas de Cyrano, ce «tiers inclus» est le plus souvent la cible qu'on entend ridiculiser ou brutalement démolir. Or, le statut des Lettres de Cyrano s'embrouille et se complexifie puisqu'elles sont d'une part fictives (le statut du destinateur des Lettres se démultipliant à l'infini sans se cantonner à la seule persona de l'auteur - Cyrano adopte différentes signatures et dédouble les points de vue ${ }^{3}$ ) et qu'elles n'ont eu, d'autre part, que la

XVIIe siècle : ses tensions, ses questions, ses excès clandestins, ses incertitudes sombres ou joyeuses » (2004, p. 47).

3 En effet, les Lettres sont diversement signées. Dans la majorité des cas, même, la signature patronymique n'est pas majoritaire, laissant plutôt place à des formulations générales ("votre serviteur», "votre très humble», «votre esclave», etc.). Mathilde Lévesque considère que «l'auteur peut choisir lui-même les lettres dont il se réclame en tant que personne physique, et il le manifeste en 
publication comme horizon éditorial (c'est-à-dire que les lettres n’ont jamais circulé jusqu'aux prétendus destinataires). Par conséquent, en arrachant la lettre de sa pratique courante et familière, Cyrano met en exergue au moins trois choses. D'abord, le phénomène épistolaire s'entend régulièrement, sous l'Ancien Régime, comme une oscillation entre le dialogue et le monologue, comme une négociation entre une présence et une absence. En désinvestissant la lettre de son contexte foncier de communication, Cyrano aménage un dispositif spéculaire, où il parle de lui à lui-même, si bien que toute possibilité d'échange se néantise d'office. Ensuite, la lettre ainsi conçue comme miroir délirant de soi-même construit une posture, une représentation de soi fondée sur une rhétorique du repli. Sans possibilité de démenti ou de contradiction, le « moi » cyranien se met à l'abri de toute attaque et de tout éventuel salissage. Enfin, l'ethos agressif mis en scène dans les Lettres entraîne un rapport pathologique à l'autre, où Cyrano procède à une déconstruction diffamée de l'ennemi.

C'est précisément l'expression ambiguë du «moi » cyranien, qui s'affirme et s'hypertrophie dans une violence discursive aux multiples entrelacements, que nous souhaitons explorer dans cet article. La méchanceté de Cyrano se décline en plusieurs modalités. Dans un premier temps, il sera question $\mathrm{du}$ contexte de répression et de censure qui surplombe et oriente la mise en écriture des Lettres, engendrant un régime de lecture que nous dirons judiciaire. Partant, Cyrano développe, au gré de ses offensives, une esthétique duelliste qui dépasse l'injonction du «soit périr, soit se venger», lieu commun

apposant son patronyme; il va de soi que dans une correspondance réelle ces arrangements n'ont pas les mêmes enjeux » (2009, p. 478). 
culturel de la querelle sous l'Ancien Régime. Enfin, la méchanceté cyranienne ainsi conceptualisée s'inscrit dans un discours de la prédation qui consiste à la fois à déshumaniser l'ennemi et à le dépourvoir de parole.

\section{Les violences libertines. De victime à bourreau}

Disons d'emblée que les Lettres sont publiées dans un contexte éditorial largement défavorable aux écrivains contestataires ou transgressifs, du moins ceux qui sont jugés tels par les autorités censoriales. Puisque les Lettres font massivement l'usage d'un "processus de déconstruction des discours d'autorité véhiculés par la culture doxale héritée» (Ronzeaud, 2006, p. 254), plusieurs stratégies de contrôle, d'autocensure et de correction sont devenues nécessaires, opérations de régie qui visent toutes, à des degrés divers, la multiplication (quasi infinie) des points de vue et des perspectives possibles. L'implication auctoriale, autrement dit, n'est jamais compromise ou révoquée par ces volte-face énonciatives. Si le regard sur le monde change parfois de focale, si les allégeances se modifient et s'effritent au gré des rééditions (Cyrano aurait tantôt été pour la Fronde, tantôt contre, de même qu'il s'affiche successivement ami et ennemi des uns et des autres ${ }^{4}$ ), ce n'est pas tant pour se déresponsabiliser d'une prise de position délétère ou certainement préjudiciable, mais bien pour accentuer l'idée selon laquelle il n'existe jamais qu'une façon unique et dogmatique de voir les choses. Cyrano met d'ailleurs le lecteur

\footnotetext{
4 Madeleine Alcover place par exemple la lettre Contre les frondeurs, dont la composition est mouvante, dans une dynamique oscillatoire, entre «louange énorme des puissances » et " critique hyperbolique de ses ennemis personnels » (1990, p. 92).
} 
explicitement en garde contre la polysémie irréductible des discours : "On ne doit pas croire toutes choses d'un homme, parce qu'un homme peut dire toutes choses. » (L, p. 85) Au fond, ce qui importe n'est pas tant l'énoncé que sa juste lecture.

Dans une lettre dédiée à une femme rousse (inconnue et réduite au statut de stéréotype), Cyrano met en abîme la présence quasi démiurgique de la censure, qui exerce un contrôle au demeurant total, surplombant et tout-puissant :

J'en étais là de ma lettre, adorable M..., lorsqu'un censeur à contresens m'arrache la plume et me dit que c'était mal se prendre au panégyrique de louer une jeune personne de beauté, parce qu'elle était rousse. Moi ne pouvant punir cet orgueilleux plus sensiblement que par le silence, je pris une autre plume, et continuai ainsi: une belle tête sous une perruque rousse, n'est autre chose que le Soleil au milieu de ses rayons ; ou le Soleil lui-même, n'est autre chose qu'un grand œil sous la perruque d'une rousse ; cependant tout le monde en médit à cause que peu de monde a la gloire de l'être ; et cent femmes à peine en fournissent une, parce qu'étant envoyées du Ciel pour commander, il est besoin qu'il y ait plus de sujets que de seigneurs. ( $L$, p. 72-73)

Le censeur reproche à Cyrano de blasphémer parce qu'il écrit l'éloge d'une femme à la chevelure rousse, figure honnie dans l'imaginaire populaire de l'Ancien Régime puisqu'elle évoquait les flammes de l'enfer et avait supposément des pouvoirs maléfiques et surnaturels. Dans un premier temps, Cyrano répond à la censure par le silence, avant de reprendre une autre plume pour démonter la mécanique de la superstition. À partir de la figure-métaphore de la femme rousse, qui agglomère les préjugés plus extravagants les uns que les autres, Cyrano dénonce l'arbitraire du rapport millénaire dominant / dominé en signalant que l'étrangeté, c'est-à-dire tout ce qui diffère de la masse commune ou bien du 
consensus, engendre toujours, chez les esprits faibles, soupçon et répression. Dans les Lettres de Cyrano, donc, on retrouve thématisés partout et diversement les dangers de l'écriture : à la fois pour celui qui tient un discours déviant et pour celui qui se montre incapable d'en déchiffrer adéquatement le sens.

Il est par ailleurs significatif de constater que les Lettres ont été annoncées en grande pompe dans la seule tragédie publiée de Cyrano, la Mort d'Agrippine, également parue en 1654. L'imprimeur s'immisce dans l'œuvre de son auteur et annonce, dans le péritexte, qu'il prépare un recueil de lettres dont la lecture serait recommandable aux beaux esprits : «Je fais rouler la Presse avec autant de diligence qu'il m'est possible pour vous en donner le contentement, \& à moy celuy de vous faire aduoüer que ie vous ay dit la verité. ${ }^{5}$ » $\mathrm{Si}$ ce procédé explicitement publicitaire n'est pas sans exemple sous l'Ancien Régime, il souligne, avant même leur publication, le caractère autant polémique que paradoxal des Lettres. Polémique, d'abord, parce qu'un régime judiciaire de lecture s'impose comme préalable : les Lettres appellent une légitimation de la part des lecteurs, qui sont les seuls dépositaires de la vérité du texte. À un livre de distance, il y a donc programmation d'une pratique de l'aveu qui est déléguée à la figure du destinataire (en dédouanant presque le destinateur de toute obligation). Ce régime de lecture est par ailleurs paradoxal, puisque la vérité dont il s'agit est proprement libertine, vérité indéfendable et qui se décline en un ensemble de propositions marginales, dangereuses et qui circulent le plus souvent de façon clandestine. Cette méthode faisant du lecteur un agent actif du texte est mimétique du «déniaisement»

5 Cyrano de Bergerac, «Le libraire au lecteur », dans La Mort d'Agrippine, Paris, Charles de Sercy, 1654, non paginé. 
libertin, phénomène pendant lequel un individu s'affranchit des discours d'autorité jugés mensongers et trompeurs. Pour Cavaillé, le déniaisement libertin est une remise en cause à la fois «[d]es croyances et [d]es dogmes religieux ainsi que [de] la vérité et [de] la validité de la morale sexuelle de la société chrétienne » (2013, p. 21), remise en question qui passe par un jeu chronique sur les formes de l'imposture. C'est par l'expérience, qui plus est par une expérience essentiellement herméneutique, que l'esprit anciennement crédule se défait de ses superstitions. C'est à pareil travail thérapeutique sur le lecteur que s'appliquent les Lettres de Cyrano, qui engagent une conversation, voire une éventuelle conversion, l'un des enjeux des Lettres étant évidemment d'emporter l'adhésion des lecteurs, transfigurés en juges et témoins.

Si les Lettres programment un régime de lecture fondé sur le déniaisement, elles constituent aussi, à bien des égards, une réflexion sur les principes mêmes de la communication et des possibles du langage. La poétique épistolaire de Cyrano peut se définir comme une permanente défense et illustration de l'alternatif: la plupart des lettres sont à la fois pro et contra, si bien que nous pouvons affirmer, avec Patricia Harry, que « l'image de la réversibilité dynamique, en perpétuel mouvement, inspire l'esthétique cyranienne » (1999, p. 443). Cette dualité des prises de position s'observe notamment dans le diptyque des lettres descriptives consacrées à la fontaine d'Arcueil, que Cyrano écrit à quelques années de distance. Cette fontaine, que l'on dit magique en vertu de ses prétendus pouvoirs guérisseurs, est l'objet d'un double regard, à la fois élogieux et critique. Cette double description s'accompagne d'un paratexte dévoilant la poétique erratique ou la logique constamment contradictoire qui gouverne la composition des Lettres. Le «je» narrateur se 
montre d'une ambivalence toute libertine, pouvant tour à tour adopter des opinions contraires et irréconciliables :

Cette lettre d'Arcueil ayant été perdue, l'auteur longtemps après en fit une autre : mais comme il ne se souvenait presque plus de la première, il ne rencontra pas les mêmes pensées. Depuis, il retrouva la perdue, et comme il est assez ennemi du travail, il ne crut pas que le sujet fût digne d'épurer chaque lettre, en ôtant de chacune les imaginations qui se pourraient rencontrer dans l'autre. ( $L$, p. 59)

Cyrano évacue l'idée d'une fixation durable du sens. Le contenu même de la lettre, sujet aux variations les plus brusques, n'apparaît plus premier. En fait, il faut chercher audelà de l'objet même de la lettre : c'est la forme, vindicative et outrée, qui l'emporte en définitive. Les lettres seraient donc une façon d'accueillir une indignation généralisée, qui se démultiplie à l'envi selon les objets considérés. Les Lettres n'informent pas tant d'un contenu qu'elles performent, de façon itérative, une attitude belliqueuse et violente. Ce qui prime ainsi dans les Lettres n'est pas exactement leur énoncé, vacillant et fuyant par définition, que leur énonciation. Elles ne mettent pas en avant des vérités dogmatiques ou péremptoires, mais révèlent plutôt un processus : celui qui permet de déconstruire les discours hypocrites que tiennent les autorités politiques et religieuses. Ce faisant, Cyrano construit son rapport au monde en termes de dissociation et de rupture, pavant ainsi la voie à une esthétique « duelliste».

\section{L'esthétique duelliste. Les mots " escrimeurs »}

Il importe de constater, dans les Lettres, la récurrence d'un motif dédoublé et modulé à l'infini : celui du duel. Si la querelle, 
sous l'Ancien Régime, est prioritairement discursive, elle renvoie néanmoins, par sa dimension violente et judiciaire, à la pratique du duel héritée de l'époque féodale. Le duel se définit notamment comme une manifestation pathogène de l'honneur, qui est l'un des fondements matriciels de la cohésion sociale, du moins dans les sociétés aristocratiques. Pour Hervé Drévillon, «l'honneur est une injonction, une obligation impérieuse, permettant à l'individu qui s'y soumet de s'affirmer comme sujet, dans la double acception d'individu assujetti et de personnalité souveraine» (2002, p. 364). Au cœur de la pratique du duel se trouve aussi l'idée de la recherche de la gloire la plus grande : la bravoure au combat permet à la fois de se rendre justice soi-même, en s'égalant en puissance aux pouvoirs divin et monarchique, et d'ajouter des fleurons à sa réputation personnelle. Conçu comme un contrat antisocial, le duel, dans la société de l'Ancien Régime, est une forme, violente et illicite, de régulation sociale. Dans les Lettres cyraniennes, un rapprochement explicite s'opère effectivement entre duel physique et querelle littéraire, à telle enseigne que la plume et l'épée s'assimilent bientôt l'une à l'autre :

Quoique je me porte en homme qui crève de santé, je ne laisse pas d'être malade depuis trois semaines que ma philosophie est tombée à la merci des gladiateurs: je suis incessamment travaillé de la tierce et de la quarte. J'aurais perdu la connaissance du papier, si les cartels s'écrivaient sur autre chose ; je ne discerne déjà plus l'encre d'avec le noir à noircir ; et enfin, pour vous faire réponse, j'ai presque été forcé de vous écrire avec mon épée, tant il est glorieux d'écrire mal parmi des personnes dont les plumes ne se taillent point. ( $L$, p. 97)

Sans doute faut-il remarquer que la querelle au XVII siècle, même si elle élit la littérature comme champ de bataille, ne pourrait se dénouer sans l'apparence d'une répartition des 
forces : il faut coûte que coûte qu'il y ait un vainqueur. La lettre "Le Duelliste», de laquelle est tiré cet extrait mettant en exergue une certaine éthique de l'antagonisme, verse dans la démesure : Cyrano y prétend qu'il est assiégé par tellement de demandes de duels qu'il n'aurait dans le monde qu'ennemis et persécuteurs. Dans cette perspective à la fois pessimiste et presque paranoïaque, Cyrano écrit comme d'autres guerroient : il défend sa place, protège son honneur et vilipende ses ennemis dans un jeu de massacre.

Or, prendre part à un duel littéraire n'engage pas les mêmes dispositifs et la même scénographie qu'un combat à mains armées. D'évidence, les conditions de victoire diffèrent considérablement : si le plus fort, le plus rapide et le plus rusé l'emporte dans une confrontation physique, il faut plutôt s'en remettre à autrui dans un conflit qui se joue tout entier sur le papier. Comme Alain Viala le remarque à juste titre, toute "querelle se spécifie comme une plainte portée devant une instance de jugement, qui peut être le public» (2013, p.11). C'est en effet la communauté de lecteurs appréciant à distance le combat qui en déterminera l'issue. Bref, puisque la lettre est un objet public, qui s'inscrit dans un espace discursif particulier, faisant intervenir différents agents, idéologiquement et culturellement circonscrits, la fin des hostilités demeure toujours liée à son contexte de diffusion.

Étant donné que les querelles initiées ou poursuivies par Cyrano n'engagent pas des discours de vérité, mais toujours des discours de légitimation, ce sera le lecteur qui, en dernière instance, agira à titre de juge et partie. C'est dans cet horizon lectorial que Cyrano développe ce que nous pourrions appeler ses conditions de victoire. S'il est bien un homme de son époque 
en déclarant à demi sérieux que "l'honneur sali ne se lave qu'avec du sang » (L, p. 103), Cyrano se demande s'il n'y a pas des solutions intermédiaires et moins catégoriques que l'assassinat qui permettraient de sauver les apparences :

Non, non, je ne dédaigne point, c'est craindre son ennemi, de vouloir par le moyen de la mort, ou l'éloigner de soi, ou s'éloigner de lui : pour moi, je n'appréhende pas qu'il soit où je serai. Il tient à gloire de n'avoir jamais redouté les Parques : s'il veut que je le croie, qu'il se tue, j'irai consulter tous les sages pendant soixante ou quatre-vingts ans, pour savoir s'il a bien fait [...]. (L, p. 103-104)

Cette manière burlesque de concevoir l'issue d'un duel par le suicide de celui qui nous méprise constitue évidemment une entorse au rituel fortement normé de l'époque, en déplaçant l'odieux de la preuve du côté de l'offenseur. S'il se dit prêt à mourir pour préserver sa réputation, Cyrano suggère que l'offenseur s'en prenne plutôt à lui-même en plaçant sa propre existence en gage. Cyrano échafaude une hiérarchie où être humilié publiquement - en ayant la possibilité de se défendre - constitue un moindre mal que de risquer bêtement la mort. Au-delà de la stricte ironie ou de l'inversion des mécanismes de l'affrontement, cette conception de la vengeance refoulée accorde le primat au discours plutôt qu'au corps. En effet, la mort n'abolit pas la possibilité de dire, de calomnier ou de médire. Cyrano pense ainsi le duel dans le temps long, c'est-àdire à la fois historique et littéraire : s'il ne veut pas mourir, c'est parce qu'il juge que la meilleure attaque est discursive plutôt que physique. Les Lettres de Cyrano portent en effet la trace de cette charge à tout vent où la langue, trempée dans le vitriol, devient une arme tranchante, où le dire intempestif se veut tout à coup mortifère. 


\section{Discours de la prédation, discours de la haine?}

Chez Cyrano, la méchanceté prend volontiers les formes, sans doute convenues et attendues, de l'irritation et du fiel. Effectivement, il apparaît que la fonction primaire des Lettres cyraniennes est la dévalorisation programmée de ses adversaires et des thèses qu'ils défendent en recourant à une écriture de la véhémence, fondée essentiellement sur l'outrance, l'artifice rhétorique et le dénigrement. Ces attaques brusques et brutales, qui sont des règlements de comptes personnels ou des pointes circonstancielles, prenant par exemple nommément à partie des figures honnies et contreexemplaires (ennemis politiques ou littéraires dont il faut détruire la réputation tout en valorisant jusqu'à la boursouflure sa propre persona), s'inscrivent toutes dans une temporalité restreinte: l'accusation, même si elle est impétueuse, n'a d'autre écho que dans une stricte instantanéité. Ces agressions répétées, opérant dans le court terme, ne peuvent pas toujours se détacher du particulier pour atteindre l'universel: elles demeurent à ras de l'actualité contemporaine et traduisent dans l'immédiat une animosité qui n'a d'épaisseur que celle, éphémère et fermée, de la lettre qui la contient.

La méchanceté cyranienne ne se cantonne pas toujours à l'insulte, gratuite ou provocatrice. Au contraire, il y a dans les Lettres satiriques quelque chose comme une praxis de la méchanceté, c'est-à-dire que la violence inscrite dans chaque lettre cherche à créer un effet précis : entraîner le destinataire dans un régime d'affectivité caractérisé par l'agression et l'emportement. L'une des tensions constamment à l'œuvre dans les Lettres est effectivement celle de la responsabilisation du lecteur. C'est finalement le destinataire qui doit à la fois 
incarner et perpétuer à long terme cette force négative et destructrice. Cette dynamique du lecteur-juge emprunte beaucoup au discours juridique et aux formes procédurales, en reprenant, à nouveaux frais, les enjeux traditionnels de la polémique mais en les dévoyant. Chez Cyrano, «la polémique apparaît alors surtout comme un grand jeu de "feintise" et de fiction, renvoyant à la fois à un ethos devenu mythique (celui de l'écrivain bretteur, de l'ingenio duelliste), à une vision agonistique du cosmos, à une démultiplication des figures du sujet » (Darmon, 2004, p. 50).

Beaucoup des insultes cyraniennes sont construites selon un schéma baroque du portrait dégradé. Les attaques concernent d'abord la matérialité du corps, mais plus généralement l'appartenance ou non d'un tel au règne de l'homme. C'est en déshumanisant l'autre que Cyrano acquiert une certaine hauteur, ses ennemis étant tour à tour qualifiés de bêtes, d'insectes ou de sous-hommes. Il s'agit, en somme, d'une stratégie stéréotypée qui permet de ranger dans l'à-côté des catégories usuelles celui que l'on exècre. C'est bien de cette façon ségrégative qu'est dépeint l'auteur Charles Dassoucy, contemporain et ancien amant de Cyrano :

Non, non, petit nain, ne pensez pas être quelqu'autre chose, essayez de vous humilier en votre néant ; et croyez, comme un article de foi, que si vous êtes encore aussi petit qu'au jour de votre naissance, le Ciel l'a permis ainsi pour empêcher un petit mal de devenir grand. Enfin, vous n'êtes pas homme; et que diable êtes-vous? Vous êtes, peut-être une momie que quelque farfadet aura volée à l'École de médecine, pour en effrayer le monde. (L, p. 110)

Le portrait contrefait de Dassoucy, écrivain et musicien burlesques, a pour principale articulation l'exclusion et 
s'accomplit par le moyen de différentes stratégies de rabaissement: il est à la fois nain et momie. Au contraire des critiques massives contre les superstitions ou les impostures politiques qui essaiment dans plusieurs lettres, relevant essentiellement d'un ethos libertin (que partage paradoxalement Dassoucy), Cyrano s'attaque ici à l'un des siens avec qui il s'est récemment brouillé. La décapitation littéraire de la figure de Dassoucy est intéressante pour au moins deux raisons. D’une part, la convocation dans les Lettres de son ancien compagnon rend public un différend essentiellement privé : l'engagement contre Dassoucy s'accomplit donc sur les bases d'un pathos agressif qui supplante la solidarité libertine généralement attendue. Sans compter que le dévoilement de cette relation homosexuelle, hautement réprimée et encore taboue au XVIIe siècle, n'est pas forcément dans l'intérêt de Cyrano, comme si l'humiliation de l'autre apparaît plus importante que sa propre incrimination6. L'une des maximes du libertinage stipule en effet que l'on peut agir comme on le souhaite dans le privé, mais selon les mœurs en vigueur en public 7 . La logique qui l'emporte chez Cyrano serait celle d'une automutilation suivie d'une offensive irrésistible : à une violence victimaire et subie s'ajoute une violence active, créative, motrice. D’autre part, la stratégie employée par Cyrano, cherchant

\footnotetext{
6 Madeleine Alcover suggère que Dassoucy, dans cette querelle à trois (issue de la séparation éclatante entre les écrivains-amants Chapelle, Dassoucy et Cyrano), se place en position de victime, alors que Cyrano serait à la fois plus serein et plus brutal. En se référant à Cyrano, elle souligne que « l'esprit-fort est celui qui adhère à l'épicurisme dans sa forme la plus radicale, c'est-à-dire lucrécienne » (1999, p. 272).

7 Selon Marc-André Bernier, les libertins du XVIIe siècle ont fait leur la maxime du philosophe italien Cremonini, qui suggérait qu'une «mise en scène de la duplicité » était nécessaire pour échapper à la coercition des pouvoirs en place : "En privé, fais ce qu'il te plaît; en public, fais selon l'usage reçu.» (2001, p. 29)
} 
à rendre Dassoucy monstrueux, déformé et bestial, reproduit précisément la démarche rhétorique des détracteurs des libertins. Le discours anti-libertin (issu essentiellement des milieux jésuites) est en effet traversé par ces lieux communs déshumanisants construisant une figure pervertie et malade, qui embrasse un mode de vie dégradant (libération des mœurs) et qui adopte des idées hétérodoxes (opposition systématique aux abus de pouvoir) : «C'est par et sur la pensée de la contradiction que le libertinage fonde sa philosophie propre.» (Prévot, 2008, p. 156) Le texte méchant renvoie exactement aux mêmes mécanismes politiques de contrôle et de répression que les libertins subissent eux-mêmes : le discours du méchant ressemble tout autant à un ordre implacable qu'à une sentence. Ainsi étiqueté, Dassoucy et, plus généralement, tout ennemi à démanteler voient leur identité figée dans un discours définitoire et structurant.

Dans une autre lettre adressée à Dassoucy, le blâme s'aggrave et s'inscrit dans un registre à la fois carnavalesque et scatologique, rendant encore plus explicite la fonction de décharge de l'insulte (qui est au fond l'épandage extérieur d'une tension intérieure) :

Hé ! Par la mort, Monsieur le coquin, je trouve que vous êtes bien imprudent de demeurer en vie après m'avoir offensé : vous qui ne tenez lieu de rien au monde, ou qui n'êtes au plus qu'un clou aux fesses de la Nature; vous qui tomberez si bas, si je cesse de vous soutenir, qu'une puce en léchant la terre ne vous distinguera pas du pavé; vous enfin, si sale et si puant, qu'on doute (en vous voyant) si votre mère n'a point accouché de vous par le derrière. ( $L$, p. 112)

Il y a deux forces en jeu dans ce portrait enlaidi par la méchanceté : d'une part, restreindre l'importance de l'autre, et accroître son «moi » d'autre part. Cyrano configure donc un 
régime de singularité (qui actualise la tension entre secret, scandale et publication) où il agit à l'abri des menaces et de la violence d'État, en reproduisant, à l'échelle de la lettre, les mêmes dynamiques de combat et de diffamation qui l'assaillent par ailleurs.

C'est sans doute parce que Cyrano envisage le discours comme l'arme la plus redoutable qu'il thématise aussi massivement les vices de parole chez ses adversaires. En effet, les cibles de Cyrano sont tout à la fois aphones, verbomoteurs ou encore plagiaires. La parole (empêchée, détériorée, salie) déshumanise l'ennemi tout en le désarmant:

Souffrez donc, encore que vous prétendiez vous soustraire de l'empire que Dieu a donné aux hommes sur les bêtes, que je vous commande de vomir sur quelque chose de plus sale que mon nom, et de vous ressouvenir (car je crois que les animaux comme vous ont quelque réminiscence) que le Créateur n'a donné à ceux de votre espèce une langue que pour avaler, et non pour parler. $(L$, p. 106-107)

Cyrano, à force de rendre l'autre étranger au genre humain, empiète tout à fait sur l'univers de discours de son ennemi : l'auteur des Lettres occupe tout le champ de la parole, espace souverain de légitimation de soi et de discrimination d'autrui. Cyrano recrée un microcosme politique asymétrique où il détient seul le pouvoir de sanction et la liberté de blâmer, l'interaction étant au final doublement invalidée : d'abord parce que la lettre est fictive, ensuite parce que la victime est privée de voix.

En conclusion, Cyrano, en multipliant les insultes animalières, en réduisant systématiquement ses ennemis au statut de bête, 
s'inscrit dans un discours réitéré de la prédation. Écrire devient une véritable chasse à l'homme, fondée sur une violence quasi darwinienne, c'est-à-dire que la loi du plus fort prime. La méchanceté des Lettres constitue une stratégie d'autodéfense globale (qui engage Cyrano contre le monde), dépréciant et infériorisant l'autre afin de rétablir, sur le plan symbolique, une forme déviante de rapport de force. En effet, la posture qu'affiche Cyrano dans ses Lettres semble avoir pour principale fonction de dénoncer les impostures d'une société malade et inégalitaire en transformant une violence symbolique subie les libertins étant systématiquement pourchassés par la justice — en agression à mots armés. À force de portraits-charges, qui grossissent l'ennemi jusqu'à la contrefaçon, la lettre s'épuise, s'affadit et se dégrade en véritable cercle vicieux, où l'enjeu n'est plus d'avoir raison (la querelle étant d'abord et avant tout un conflit herméneutique dont la fixation du sens demeure le noyau), mais de rivaliser de génie, de manière parfois puérile et affectée, dans l'invention polémique ou ordurière. Une fois le dédain transformé en abjection, une fois l'esprit de contradiction inversé en goût de l'invective, le débat d'idées se métamorphose et s'enlise dans un discours de la haine fermé sur lui-même.

\section{Bibliographie}

AlCover, Madeleine. (1990), Cyrano relu et corrigé. (Lettres, Estats du Soleil, Fragment de Physique), Genève, Droz.

—. (1999), "Un gay trio : Cyrano, Chapelle, Dassoucy», dans Ralph Heyndels et Barbara Woshinsky (dir.), L'autre au XVIIe 
siècle, Tübingen, Gunter Narr Verlag, p. 265-275.

BERNIER, Marc-André. (2001), Libertinage et figures du savoir: rhétorique et roman libertin dans la France des Lumières (1734-1751), Québec, Les Presses de l'Université Laval.

CAVAillé, Jean-Pierre. (2013), Les Déniaisés. Irréligion et libertinage au début de l'époque moderne, Paris, Classiques Garnier.

CyRANO DE BERGERAC, Savinien de. (1990[1654]), Lettres satiriques et amoureuses, précédées de Lettres diverses (édit. Jean-Charles Darmon et Alain Mothu), Paris, Desjonquères.

DARMon, Jean-Charles. (2004), Le Songe libertin. Cyrano de Bergerac d'un monde à l'autre, Paris, Klincksieck.

DrÉVILlON, Hervé. (2002), "L'âme est à Dieu et l'honneur à nous. Honneur et distinction de soi dans la société d'Ancien Régime », Revue historique, vol. 2, n 654, p. 361-395.

FERREYROLLES, Gérard. (2010), "L'épistolaire, à la lettre », Littératures classiques, vol. 1, n 71, p. 5-27.

HAREL, Simon. (2008), «La méchanceté littéraire: les figures malaisées de l'autre en soi », Le Coq-Héron, vol. 1, n¹92, p. 57-69.

HARRY, Patricia. (1999), «L'altérité cyranienne : le jeu de cachecache esthético-idéologique d'un marginal fieffé», dans Ralph Heyndels et Barbara Woshinsky (dir.), L'autre au XVII siècle, Tübingen, Gunter Narr Verlag, p. 441-451.

LÉVESQUE, Mathilde. (2009), « Du manuscrit à l'édition : formes linguistiques de l'autocorrection dans les Lettres de Cyrano de Bergerac », Papers on French Seventeenth Century Literature, vol. 36, $\mathrm{n}^{\circ}$ 71, p. 475-491.

MELANÇON, Benoît. (1996), Diderot épistolier. Contribution à une poétique de la lettre familière au XVIIIe siècle, SaintLaurent/Québec, Fides. 
PRÉVOT, Jacques. (2008), «Libertinage, Cyrano, antichristianisme ", dans Hervé Bargy et Alain Mothu (dir.), Cyrano de Bergerac, Cyrano de Sannois, Turnhout, Brepols, p. 153-163.

RoNZEAUD, Pierre. (2006), «Un "proême" programmatique : les Lettres de Cyrano de Bergerac sur les saisons » dans Patricia Harry, Alain Mothu et Philippe Sellier (dir.), Dissidents, excentriques et marginaux de l'âge classique, Paris, Champion, p. 247-255.

VAN Damme, Stéphane. (2007), «Violences fondatrices? Les épreuves aux origines de l'identité libertine sous l'Ancien Régime ", article électronique, Les Dossiers du Grihl, $<$ http://dossiersgrihl.revues.org/722>.

VIALA, Alain. (2013), "Un temps de querelles », Littératures classiques, vol. 2, $\mathrm{n}^{\circ}$ 81, p. 5-22.

\section{Résumé}

Cet article se fixe l'objectif de montrer que les Lettres (1654) de Cyrano de Bergerac configurent une véritable politique de la méchanceté qui informe la fictionnalisation du «moi » cyranien. La méchanceté de Cyrano se décline en plusieurs modalités. Dans un premier temps, il sera question du contexte de répression et de censure qui surplombe et oriente la mise en écriture des Lettres, engendrant un régime de lecture judiciaire. Cyrano développe aussi, au gré de ses offensives, une esthétique duelliste qui dépasse l'injonction du « soit périr, soit se venger ", lieu commun culturel de la querelle sous l'Ancien Régime. Enfin, la méchanceté cyranienne s'inscrit dans un discours de la prédation qui consiste à la fois à déshumaniser l'ennemi et à le dépourvoir de parole. 


\begin{abstract}
This article aims to show that Cyrano de Bergerac's Lettres (1654) are built on a massive recourse of hatred, which informs his ambiguous self-fictionalization. We will first discuss the context of repression and censorship in which the Lettres were written, creating a regime of judicial reading. Cyrano is developing an aesthetic based on a vison of the world as a perpetual duel. This article also attempts to show that the theme of hatred is part of a discourse of predation where Cyrano's victims are both dehumanized and deprived of speech.
\end{abstract}

